\title{
Breast Ultra-Sound image segmentation: an optimization approach based on super-pixels and high-level descriptors
}

\author{
Joan Massich $^{a}$ and Guillaume Lemaître ${ }^{a, b}$ and Joan Martí ${ }^{b}$ and Fabrice Mériaudeau ${ }^{a}$

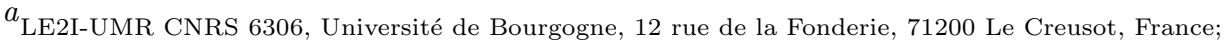

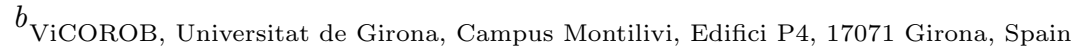

\begin{abstract}
Breast cancer is the second most common cancer and the leading cause of cancer death among women. Medical imaging has become an indispensable tool for its diagnosis and follow up. During the last decade, the medical community has promoted to incorporate Ultra-Sound (US) screening as part of the standard routine. The main reason for using US imaging is its capability to differentiate benign from malignant masses, when compared to other imaging techniques. The increasing usage of US imaging encourages the development of Computer Aided Diagnosis (CAD) systems applied to Breast Ultra-Sound (BUS) images. However accurate delineations of the lesions and structures of the breast are essential for CAD systems in order to extract information needed to perform diagnosis.

This article proposes a highly modular and flexible framework for segmenting lesions and tissues present in BUS images. The proposal takes advantage of optimization strategies using super-pixels and high-level descriptors, which are analogous to the visual cues used by radiologists. Qualitative and quantitative results are provided stating a performance within the range of the state-of-the-art.
\end{abstract}

Keywords: Breast Ultra-Sound, BI-RADS lexicon, Optimization based Segmentation, Machine-Learning based Segmentation, Graph-Cuts

\section{INTRODUCTION}

Breast cancer is the second most common cancer (1.4 million cases per year, $10.9 \%$ of diagnosed cancers) after lung cancer, followed by colorectal, stomach, prostate and liver cancers. In terms of mortality, breast cancer is the fifth most common cause of cancer death. However, it is ranked as the leading cause of cancer death among females in both western countries and economically developing countries. ${ }^{1}$

Medical imaging plays an important role in breast cancer mortality reduction, contributing to its early detection through screening for diagnosis, image-guided biopsy, treatment follow-up and suchlike procedures. ${ }^{2}$ Although Digital Mammography (DM) remains the reference imaging modality for breast cancer screening, UltraSound (US) imaging has proven to be a successful adjunct image modality. ${ }^{2}$ The main advantage of US imaging, opposed to other image modalities, lies in the discriminative power that US offers to visually differentiate benign from malignant solid lesions. ${ }^{3}$ In this manner, US screening contributes to reduce the amount of unnecessary biopsies, ${ }^{4}$ which is estimated to be between $65 \sim 85 \%$ of the prescribed biopsies, ${ }^{5}$ in favour of a less traumatic short-term screening follow-up using Breast Ultra-Sound (BUS) images. As the standard for assessing this BUS images, the American College of Radiology (ACR) proposes the Breast Imaging-Reporting and Data System (BI-RADS) lexicon for BUS images. ${ }^{6}$ This US BI-RADS lexicon is a set of standard markers that characterizes the lesions encoding the visual cues found in BUS images and facilitates their analysis. Further details regarding the US BI-RADS lexicon descriptors proposed by the ACR, can be found in this document in section 2.1.1, where visual cues of BUS images and breast structures are discussed to define feature descriptors.

All these facts show the interest in the medical community for incorporating US screening as part of the standard procedure in breast screening programs ${ }^{6}$ which encourages the development of Computer Aided Diagnosis (CAD) systems using US to be applied to breast cancer diagnosis. Building CAD systems based on the

This work was partially supported by the Regional Council of Burgundy.

Further author information, send correspondence to joan.massich@u-bourgogne.fr 
clinical tools already in use (i.e. US BI-RADS lexicon) is not straight forward. Shortcomings like the location and explicit delineation of the lesions need to be addressed, since those tasks are intrinsically carried out by the radiologists during their visual assessment of the images to infer the lexicon representation of the lesions. Therefore, developing accurate segmentation methodologies for breast lesions and structures are crucial in order to develop CAD systems that can take advantage of the already validated clinical tools.

This article proposes a highly modular and flexible framework for segmenting lesions and tissues present in BUS images. The proposal takes advantage of an energy-based strategy to perform segmentations based on discrete optimizations using super-pixels and a set of novel features analogous to the elements encoded by the US BI-RADS lexicon. ${ }^{6}$

\section{DESCRIPTION OF THE SEGMENTATION METHODOLOGY}

Optimization methodologies offer a standardized manner to approach segmentation by minimizing an applicationdriven cost function. ${ }^{7}$ Figure 1 illustrates a generic representation of the segmentation strategy here adopted to delineate breast tissues or lesions in US images. The overall segmentation can be seen as a three-steps strategy: (1) a mapping of the image into a discrete set of elements $\mathcal{S},(2)$ the optimization stage which is formulated as a metric labelling problem, and (3) a re-mapping the labels obtained from the previous stage to produce the final delineation.

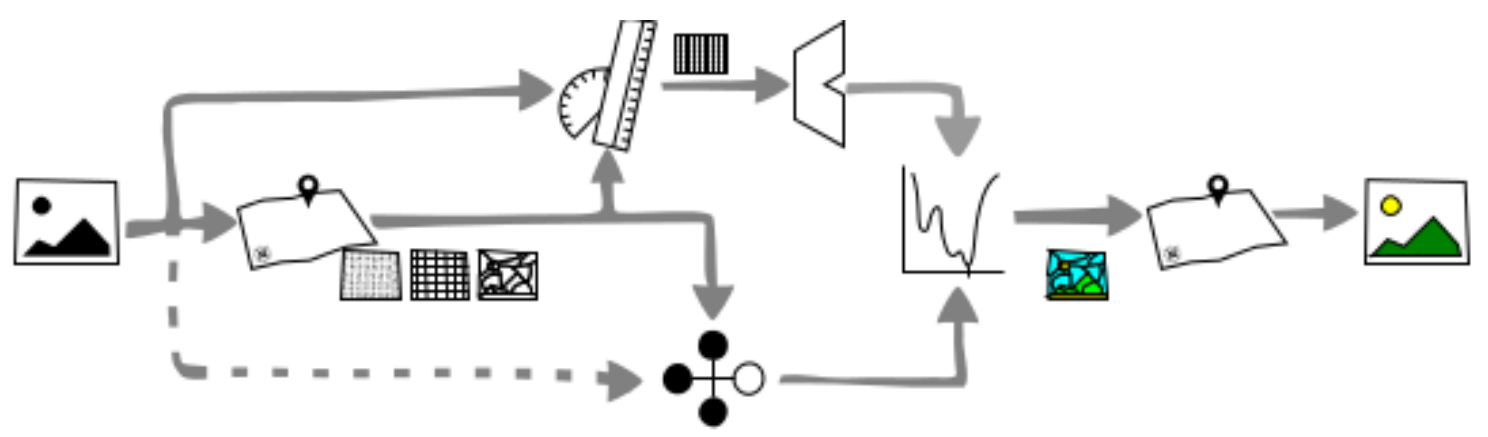

Figure 1: Conceptual block representation of the segmentation methodology

In order to formulate the segmentation like a metric labelling problem, the image is conceived as a discrete set of elements $\mathcal{S}$ that need to be labelled using a label $l$ from the labelling set $\mathcal{L}$ (i.e. $\mathcal{L}=\{$ lesion, $\overline{\text { lesion }}\}$ or $\mathcal{L}=\{$ lungs, fat, $\cdots$, lesion $\}$ ). Let $\mathcal{W}$ be all the possible labelling configurations of the set $\mathcal{S}$, given $\mathcal{L}$. Let $U(\cdot)$ be a cost function encoding the goodness of the labelling configuration $\omega \in \mathcal{W}$ based on the appearance of the elements in $\mathcal{S}$, their inner relation and some designing constraints. Then, the desired segmentation $\hat{\omega}$ corresponds to the labelling configuration that minimizes this cost function, as described in Equation (1).

$$
\hat{\omega}=\arg \min _{\omega} U(\omega)
$$

It is worth to mention here that not all the minimization strategies are applicable or adequate to find $\hat{\omega}$. The convenience of a particular minimization strategy is determined by the nature of $U(\cdot)$ and $\mathcal{W}$ (see section 2.3).

This goodness measure $U(\cdot)$ must be defined to take into account the appearance of the target region, its relation with other regions and other designing constraints. Equation (2) describes this cost function as the combination of two independent costs that need to be simultaneously minimized as a whole.

$$
U(\omega)=\sum_{s \in s} D_{s}\left(\omega_{s}\right)+\sum_{s} \sum_{r \in \mathcal{N}_{s}} V_{s, r}\left(\omega_{s}, \omega_{r}\right)
$$

Where, the left hand side of the expression integrates the so-called data term, while the right hand side integrates the pairwise term, which is also referred as the smoothing term. Both terms are shaped by $\mathcal{S}$ and 


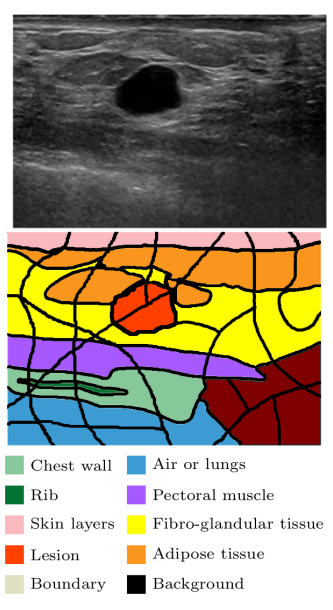

(a) Problem definition
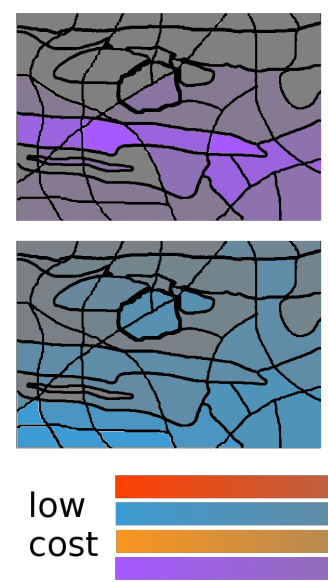
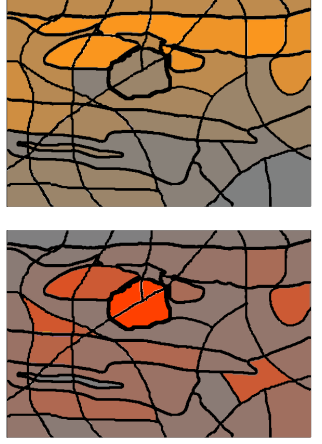

High Cost

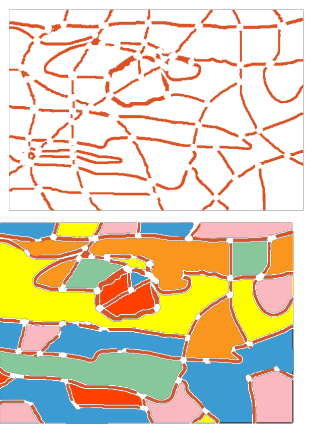

low cost
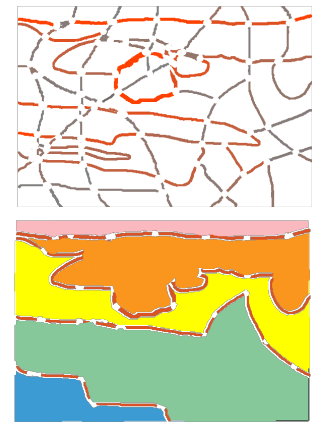

High

(b) Data term

Figure 2: Methodology terms interpretation

evaluated in the labelling space $\mathcal{W}$. Figure 2 uses the problem of delineating the tissues present in a BUS image to represent the working principles of the data and pairwise terms in eq. (2).

In general, $\mathcal{S}$ can be any discrete set representing the image (i.e. pixels, overlapping or non overlapping windows, etc.). For this work $\mathcal{S}$ is chosen to be a super-pixels representation of the image. Super-pixels can be seen as the output of an over-segmentation process or as a set of pixel collections that are contiguous and coherent with respect to a given metric. Either way, super-pixels are no overlapped irregular groups of similar connected pixels. ${ }^{8}$ Figure 2a illustrates one such representation $\mathcal{S}$, applied to a BUS image example. The superpixels are coloured according to the image's Ground Truth (GT). For the rest of this work, $\mathcal{S}$ is considered to be the super-pixels resulting from an over-segmentation of the image using Quick-shift. ${ }^{9}$

Bear in mind that given an unseen BUS image, the ultimate goal is to represent the image as a set of superpixels and infer the appropriated labelling for each of them. This goal requires to define: a data term, a pairwise term, and a proper minimization methodology.

\subsection{The data term}

Given a label configuration $\omega \in \mathcal{W}$, the data term penalizes the labelling of a particular image element or site $\left(\omega_{s}=l\right)$ based on the data associated to $s$. In this manner, $D_{s}\left(\omega_{s}=l_{\checkmark}\right)<<D_{s}\left(\omega_{s}=l_{\chi}\right)$. Figure 2b illustrates the data cost associated to some arbitrary labelling configurations to clarify the desired effect (or behaviour) of this data term (fig. 2a shows the GT of each site $s$ ). Notice that the labelling configurations $\omega$ used in fig. 2b have the particularity that all sites share the same label, $\omega \in\left\{\omega_{s}=l, \forall s \in \mathcal{S}\right\}$.

Designing an obscure heuristic to comply with the desired behaviour of $D(\cdot)$ out of the box, is rather a complicated task. Therefore, an easier and cleaner approach is to take advantage of Machine Learning (ML) techniques to design this data cost in a systematic manner based on a training stage. The idea is to generate a data model for each label (or class) in $\mathcal{L}$ from training samples, and let $D(\cdot)$ be a distance or goodness measure reflecting the likelihood for $s$ to belong to class $l$. ML offers a systematic but flexible approach to customize $D(\cdot)$. Different features to represent the data, custom construction of the data models by using different classifiers, training techniques or including arbitrary constraints; can be used to achieve the desired data term without changing the overall scheme.

Despite details regarding the construction $D(\cdot)$ are out of the scope of this report, the rest of this section 2.1 summarizes this process. For further details the reader is referred to Massich et al. ${ }^{9}$ The usage of ML as part of the proposed framework to determine $D(\cdot)$ is represented at the upper side of the diagram in fig. 1, which can be divided into two blocks: (a) the features to represent the samples, and (b) the tools to encode $D(\cdot)$ based on the features and the training. 


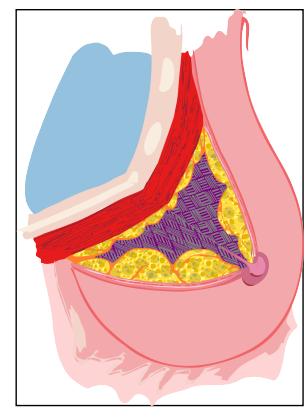

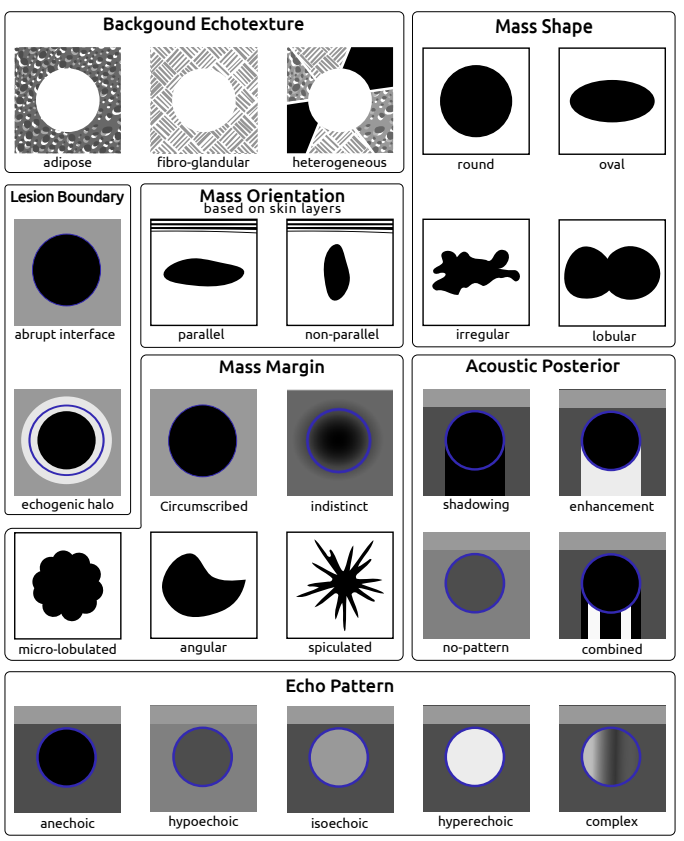

(b) Breast lesion characteristics in US screening influencing clinical management ${ }^{6}$

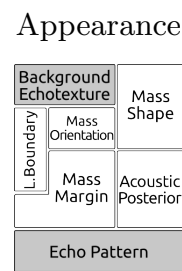

Atlas, Brightness

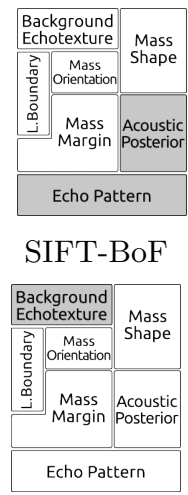

(c) Visual cues encoded in the features

(a) Breast structure

Figure 3: Visual reference of breast structures and visual cues used for standard BUS image assessment and diagnosis.

\subsubsection{BUS features to build the data term}

Figure 3 is a three-parts illustration to graphically summarize the visual cues that can be found in BUS images and their incorporation to the data term. The structures of the breast and their rendering when using a handheld 2D US probe are shown in fig. 3a. Figure 3b illustrates the lexicon proposed by the ACR. ${ }^{6}$ Whereas fig. $3 \mathrm{c}$ relates the visual cues to the following features:

Appearance Based on the multi-labelled GT, a Median Absolute Deviation (MAD) histogram model for every tissue label is built. The Appearance feature is computed as the $\chi^{2}$ distance between histogram of $s$ and the models.

Atlas Based on the multi-labelled GT an atlas is build to encode the label likelihood based on the location of $s$.

Brightness Takes an intensity descriptor of $s$ (i.e: mean, median, mode) and compares it with some intensity markers of the set $\mathcal{S}$ such as the minimum intensity value, the maximum, its mean, etc.

Self-Invariant Feature Transform (SIFT)-Back-of-Features (BoF) $s$ is represented as the occurrences of a SIFT dictionary of 36 words. ${ }^{10}$

In order to incorporate multi-resolution, each super-pixel is group with its adjacent super-pixels such that $s^{\prime}=\left\{s \cup \mathcal{N}_{s}\right\}$, the features are recalculated using $s^{\prime}$ and concatenated to the original feature descriptor of $s$. This operation can be repeated several times.

Notice that not all the visual cues compiled by the lexicon are suitable to build the data term. Mass shape, orientation and margin cues do not characterize an individual super-pixel but a group of contiguous super-pixels sharing the same label. Characterising super-pixels in those terms imply that super-pixels should be image elements of the same order as the lesions. This is not the case, since the bottom-line is to aggregate small regions to form the segmentation. The lesion boundary cue falls at the other extreme. To encode this visual cue as data term feature implies to use smaller super-pixels in order to be fully contained within the halo, limiting the discriminatory power of other features that need larger regions to build higher-level descriptors. 
In this manner the B.Echo-texture, is mainly encoded by the SIFT-BoF but also through the Appearance feature since elements would have the same model. Acoustic Posterior is mainly captured by the Brightness feature, but the Atlas also brings crucial information to compensate for intensity inhomogeneities caused by signal attenuation present in the most posterior parts of the image. The Echo Pattern cue, encodes the echogenity of a region with respect to the adipose tissue. This can be found at the anterior part of the image with its echogenity close to the middle of the spectrum. Appearance, Brightness and the Atlas account for such information.

\subsubsection{The data term construction}

For the work here reported, a Radial Basis Function (RBF)-Support Vector Machines (SVM) classifier is used to determine the data model during the training stage. During the testing stage, $D_{s}\left(\omega_{s}=l\right)$ corresponds to the distance between the testing samples and the model associated to $l$ as the SVM classification reward.

\subsection{The pairwise (or smoothing) term}

The pairwise term represents the cost associated to $\omega_{s}$ taking into account the labels of its neighbour sites, $\omega_{r}$, $r \in \mathcal{N}_{s}$. This term models a Markov Random Fields (MRFs) or a Conditional Random Fields (CRFs). The typical form of this term, given in eq. (3), is called homogenization which acts as a regularization factor favouring configurations that have a coherent labelling.

$$
V_{s, r}\left(\omega_{s}, \omega_{r}\right)= \begin{cases}\beta, & \text { if } \omega_{s} \neq \omega_{r} \\ 0, & \text { otherwise }\end{cases}
$$

Figure 2c offers a visual interpretation of this cost. The more fragmented is the segmentation $\omega$, the higher the overall pairwise term; since every boundary brings a penalization $\beta$ to the total cost $U(\omega)$. In this manner the regularization term can be seen as a post-processing or denoising stage since that some sites will flip their labelling if the cost of fragmenting the regions is larger than the cost of adopting their neighbour's label.

More sophisticated smoothing terms, where boundaries have different penalization based on site relations in $\mathcal{S}$ and image information as illustrated in fig. 2c, are also naturally handled by the proposed framework. ${ }^{9}$

\subsection{Searching the best labelling configuration}

Once defined $U(\omega)$ so that the cost for a particular labelling configuration $\omega$ can be computed, the problem of finding $\hat{\omega}$ corresponding to the global minimum of the space $\mathcal{W}$ of all possible labelling configurations needs to be faced.

This problem falls into the category of NP-hard problems. Moreover, due to limitations in building $U(\cdot)$ such as noise, training policies, etc. there are no guarantees that the global minimum $\hat{\omega}$ corresponds to the true labelling.

Nevertheless, there is a large body of literature proposing methodologies to find suboptimal solutions to the problem trading-off between time of convergence and accuracy of the solution reached. Szeliski et al. ${ }^{11}$ conducted an exhaustive review in terms of solution quality and runtime of the most common energy minimization algorithms used in Computer Vision (CV), such as Iterated Conditional Modes (ICM), Simulate Anealing (SA) or Graph-Cuts (GC).

The minimization strategy used for this work is GC. This technique was initially introduced to solve CV applications by Boykov et al. ${ }^{12}$ Soon after its introduction, it becomes the minimization technique of choice for CV problems. Since, when GC is applicable, it allows to rapidly find a strong local minima guaranteeing that no other minimum with lower energies can be found. ${ }^{13} \mathrm{GC}$ is applicable if, and only if, the pairwise term favours coherent labelling configurations and penalizes labelling configurations where neighbours labels differs; such is our case, given in eq. (3). 


\section{METHOD EVALUATION AND COMPARISON}

A 16 BUS images dataset with accompanying multi-label GT delineating all the structures present in the images has been used to evaluate the proposed methodology for lesion segmentation application. Every image in the dataset presents a single lesion with variable extension. The size of the lesions ranges from under 1/100 to over $1 / 5$ of the image. The dataset composed of cysts, Fibro-Adenomas (FAs), Ductal Inflating Carcinomas (DICs) and Inflating Lobular Carcinomas (ILCs).

Figure 4 shows qualitative results, results whereas the quantitative results from the best configuration are reported as a table in fig. 5b. ${ }^{9}$ Notice that for the proposed framework, the performance in terms of Area Overlap (AOV) is limited by the capacity of the super-pixels to snap the desired boundary. Figure 4b shows how the delineation resulting from a proper labeling of the super-pixels differs from the GT. Despite the fact that a False Positive Ratio (FPR) of 0.4 seems significant, based on our experiments most of the images produce no False Positive (FP) lesions. However, images with FP lesions, are likely to produce more several of them as shown in fig. 4c-e. The amount of FP lesions can be trimmed by applying a higher cost in the pairwise therm (compare fig. 4d and fig. 4e. Nevertheless, a severe increasing of the pairwise cost also increases the False Negative Ratio (FNR) since some lesions are missed due to over-smoothing. ${ }^{9}$ The situation of having a larger FNR is less desirable than reducing the FPR. The FNR reported in fig. 5b is caused by an image within the dataset that its lesion is fully contained in a single super-pixel and still around $20 \%$ of this super-pixel's area is healty tissue.

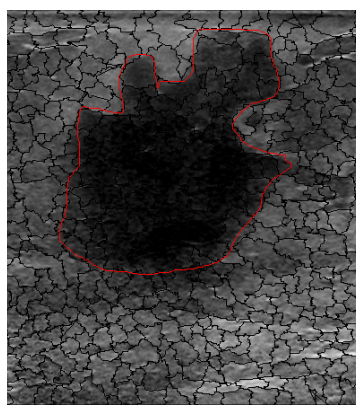

(a)

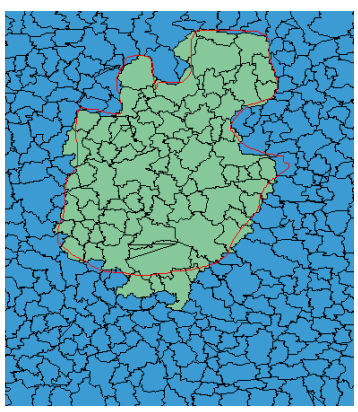

(b)

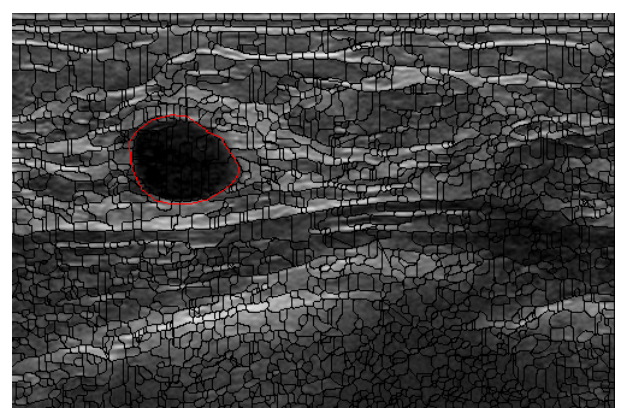

(c)

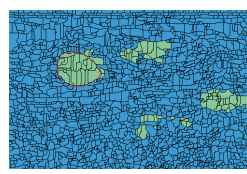

(d) weak $V(\cdot, \cdot)$

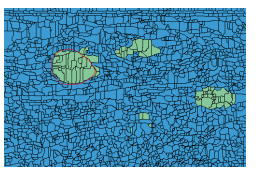

(e) strong $V(\cdot, \cdot)$

Figure 4: Qualitative results. (a) Example 1: orignal image, super-pixels' delineations and GT. (b) Differences between GT and the delination resulting from super-pixels' boundary. (c) Ex. 2. (d, e) Pairwise term influence to FPR, FNR

Due to the lack of publicly available data (and code) there is no manner to perform a methodology comparison further than compiling the results reported in the literature. The table in Figure 5a complies the evaluation reported by the authors of the most relevant methodologies found in the literature. ${ }^{9}$ Details about the methodologies proposed in the literature can also be found in the aforesaid table. Specifically it is detailed the category of technique been used for detecting the lesions, segmenting it and post-process the delineations (if any). The studied categories are: ML, Active Contour Model (ACM) and others. The iconography used in fig. 5a also illustrates if those stages are treated as independent and connected in a daisy-chain fashion, or otherwise the stages are addressed in an atomic manner.

Figure $5 \mathrm{c}$ renders the information present in fig. 5a and fig. 5b in a visual manner compare all the results at once. The methodologies arranged in a radial fashion and grouped by its most representative technology category. In red it can be found a small, medium and large categorization of the dataset reported for testing. The concentric circles represent AOV. The blue line correspond to the AOV results reported in the literature, whereas the black line indicates the AOV our framework scored in our testing. An extra element is also represented in fig. $5 \mathrm{c}$ as blue swatch delimited by two blue dashed lines. The boundaries of this swatch correspond to performance of expert radiologists in terms of AOV based on an inter- and intra-observer experiment carried out by Pons et al. ${ }^{14 *}$.

${ }^{*}$ The dataset used for testing the framework here proposed corresponds to the subset of images used by Pons et al. ${ }^{14}$ that have accompanying multi-labelled GT. 
When comparing the results it is clear the inconvenience of unexciting public data, since several of the results outperform the manual delineations studied in. ${ }^{14}$ It can also be seen that the category tested in larger datasets is ML, whereas ACM lead to better segmentations since the lesion boundary is easier to model in ACM compared to ML based techniques.

\begin{tabular}{|c|c|c|c|c|c|c|c|c|c|c|c|c|c|c|c|c|}
\hline Method Id ${ }^{[r e f]}$ : & $\mathrm{a}^{15}$ & $\mathrm{~b}^{16}$ & $c^{17}$ & $\mathrm{~d}^{18}$ & $\mathrm{e}^{19}$ & $\mathrm{f}^{20}$ & $\mathrm{~g}^{21}$ & $\mathrm{~h}^{22}$ & $\mathrm{i}^{23}$ & $\mathrm{j}^{24}$ & $\mathrm{k}^{25}$ & $1^{26}$ & $\mathrm{~m}^{27}$ & $\mathrm{n}^{28}$ & $\mathrm{o}^{29}$ & $\mathrm{p}^{30}$ \\
\hline $\begin{array}{l}\text { Dataset size: } \\
\text { techonlogy used for: } \\
\text { detection } \\
\text { segmetnation } \\
\text { post-processing }\end{array}$ & 76 & 20 & 32 & 20 & 82 & 480 & $\begin{array}{l}347 \\
8\end{array}$ & 352 & 25 & 120 & 6 & 400 & 50 & 20 & 118 & 웅 \\
\hline AOV (in \%): & 88.1 & 86.3 & 88.3 & 85.2 & 62.0 & 75.0 & 84.0 & 54.9 & 64.0 & 83.1 & 73.3 & 73.0 & 85.0 & 78.6 & 77.6 & 74.5 \\
\hline
\end{tabular}

(a) BUS images lesion segmentation strategies compiled from the bulk of the literature: reported quantitative results and methodology highlights.

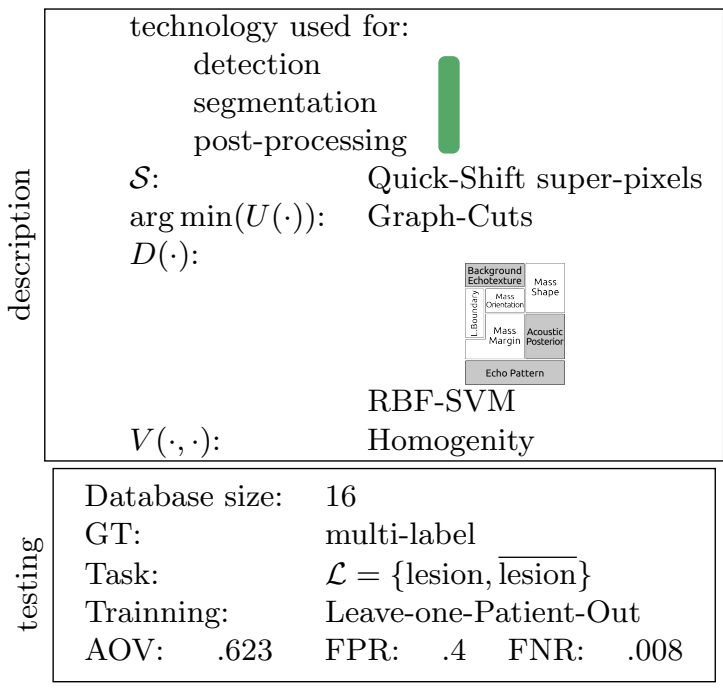

(b) Higlights of the proposed methodology

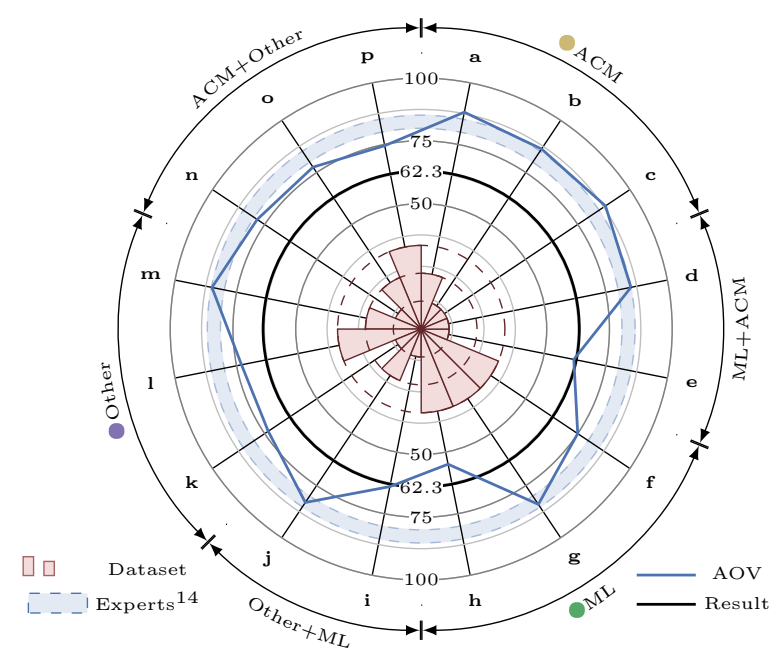

(c) comparison

Figure 5: Quantitative results compilation and comparison

\section{CONCLUSIONS}

This work presents a segmentation strategy to delineate lesions in BUS images using an optimization framework that takes advantage of all the facilities available when using ML techniques. Despite the limitation that the final segmentation is subject to the super-pixels' boundaries, the AOV results here reported are similar to those reported by other methodologies in the literature. A higher AOV result can be achieved by deforming the delineation resulting from the proposed framework using this to initialize a second post-processing step based on ACM. In this manner the contour constrains could be applied to achieve a more natural delineation.

\section{REFERENCES}

1. A. Jemal et al., "Global cancer statistics," CA: A Cancer Journal for Clinicians 61, 2011.

2. R. A. Smith et al., "American cancer society guidelines for breast cancer screening: update 2003," CA: a cancer journal for clinicians 53(3), pp. 141-169, 2003.

3. A. T. Stavros, D. Thickman, C. L. Rapp, M. A. Dennis, S. H. Parker, and G. A. Sisney, "Solid breast nodules: Use of sonography to distinguish between benign and malignant lesions," Radiology 196(1), pp. 123-34, 1995.

4. S. Ciatto, M. Rosselli del Turco, S. Catarzi, D. Morrone, et al., "The contribution of ultrasonography to the differential diagnosis of breast cancer.," Neoplasma 41(6), p. 341, 1994. 
5. Y. Yuan, M. L. Giger, H. Li, N. Bhooshan, and C. A. Sennett, "Multimodality computer-aided breast cancer diagnosis with ffdm and dce-mri.," Academic radiology 17(9), p. 1158, 2010.

6. E. Mendelson, J. Baum, B. WA, et al., BI-RADS: Ultrasound, 1st edition in: D'Orsi CJ, Mendelson EB, Ikeda DM, et al: Breast Imaging Reporting and Data System: ACR BIRADS - Breast Imaging Atlas, American College of Radiology, 2003.

7. D. Cremers, M. Rousson, and R. Deriche, "A review of statistical approaches to level set segmentation: integrating color, texture, motion and shape," International journal of computer vision $\mathbf{7 2}(2), 2007$.

8. R. Achanta et al., "SLIC superpixels compared to state-of-the-art superpixel methods," 2012.

9. J. Massich, Deformable object segmentation in ultra-sound images. PhD thesis, University of Girona, and University of Burgundy, december 2013.

10. J. Massich et al., "Sift texture description for understanding breast ultrasound images," in Breast Imaging, Lecture Notes in Computer Science 8539, pp. 681-688, Springer International Publishing, 2014.

11. R. Szeliski et al., "A comparative study of energy minimization methods for markov random fields with smoothness-based priors," Pattern Analysis and Machine Intelligence 30(6), pp. 1068-1080, 2008.

12. Y. Boykov, O. Veksler, and R. Zabih, "Fast approximate energy minimization via graph cuts," Pattern Analysis and Machine Intelligence, IEEE Transactions on 23(11), pp. 1222-1239, 2001.

13. A. Delong, A. Osokin, H. N. Isack, and Y. Boykov, "Fast approximate energy minimization with label costs," International Journal of Computer Vision 96(1), pp. 1-27, 2012.

14. G. Pons, J. Martí, R. Martí, S. Ganau, J. Vilanova, and J. Noble, "Evaluating lesion segmentation in breast ultrasound images related to lesion typology," Journal of Ultrasound in Medicine, 2013.

15. B. Liu, H. D. Cheng, J. Huang, J. Tian, X. Tang, and J. Liu, "Probability density difference-based active contour for ultrasound image segmentation," Pattern Recognition, 2010.

16. L. Gao, X. Liu, and W. Chen, "Phase- and GVF-Based level set segmentation of ultrasonic breast tumors," Journal of Applied Mathematics 2012, pp. 1-22, 2012.

17. M. Alemán-Flores, L. Álvarez, and V. Caselles, "Texture-oriented anisotropic filtering and geodesic active contours in breast tumor ultrasound segmentation," J Math Imaging Vis 28(1), pp. 81-97, 2007.

18. Q.-H. Huang, S.-Y. Lee, L.-Z. Liu, M.-H. Lu, L.-W. Jin, and A.-H. Li, "A robust graph-based segmentation method for breast tumors in ultrasound images," Ultrasonics 52(2), pp. 266-275, 2012.

19. A. Madabhushi and D. Metaxas, "Combining low-, high-level and empirical domain knowledge for automated segmentation of ultrasonic breast lesions," IEEE Transactions on medical imaging, 2003.

20. Z. Hao, Q. Wang, Y. K. Seong, J.-H. Lee, H. Ren, and J.-y. Kim, "Combining CRF and multi-hypothesis detection for accurate lesion segmentation in breast sonograms," in Medical Image Computing and ComputerAssisted Intervention-MICCAI 2012, pp. 504-511, Springer, 2012.

21. J. Zhang, S. K. Zhou, S. Brunke, C. Lowery, and D. Comaniciu, "Database-guided breast tumor detection and segmentation in 2D ultrasound images," in SPIE Medical Imaging, 7624, 2010.

22. G. Xiao, M. Brady, J. A. Noble, and Y. Zhang, "Segmentation of ultrasound B-mode images with intensityinhomogeneity correction," IEEE Transactions on medical imaging 21(1), pp. 48-57, 2002.

23. J. Massich, F. Meriaudeau, E. Pérez, R. Martí, A. Oliver, and J. Martí, "Lesion segmentation in breast sonography," Digital Mammography, pp. 39-45, 2010.

24. J. Shan, H. D. Cheng, and Y. Wang, "Completely automated segmentation approach for breast ultrasound images using multiple-domain features," Ultrasound in Medicine 6 Biology 38(2), pp. 262-275, 2012.

25. C. Yeh, Y. Chen, W. Fan, and Y. Liao, "A disk expansion segmentation method for ultrasonic breast lesions," Pattern Recognition, 2009.

26. K. Horsch and other, "Automatic segmentation of breast lesions on ultrasound," Medical Physics, 2001.

27. W. Gómez et al., "Computerized lesion segmentation of breast ultrasound based on marker-controlled watershed transformation," Medical Physics 37(1), p. 82, 2010.

28. Y.-L. Huang and D.-R. Chen, "Automatic contouring for breast tumors in 2-D sonography," in Engineering in Medicine and Biology Society, 2005. IEEE-EMBS 2005, pp. 3225-3228, IEEE, 2006.

29. Y.-L. Huang, Y.-R. Jiang, D.-R. Chen, and W. K. Moon, "Level set contouring for breast tumor in sonography," Journal of digital imaging 20(3), pp. 238-247, 2007.

30. J. Cui, B. Sahiner, H.-P. Chan, A. Nees, et al., "A new automated method for the segmentation and characterization of breast masses on ultrasound images," Medical Physics 36(5), p. 1553, 2009. 